\title{
On the Modal Understanding of Triadic Contexts
}

\author{
Frithjof Dau and Rudolf Wille \\ Technische Universität Darmstadt, Fachbereich Mathematik \\ Schloßgartenstr. 7, D-64289 Darmstadt, \{dau,wille\}@mathematik.tu-darmstadt.de
}

\begin{abstract}
A triadic context consists of sets of formal objects, formal attributes, and formal conditions together with the formalization of the ternary relation saying when an object has an attribute under a certain condition. The modal understanding of necessity and possiblity occurs naturally in triadic contexts, especially when the dyadic relationships between formal objects and attributes are considered: a formal object $g$ has "necessarily" a formal attribute $m$ if $g$ has $m$ under all formal conditions of the context; $g$ has "possibly" $m$ if $g$ has $m$ under some formal condition. Such necessity and possibility relations give rise to dyadic contexts allowing a modal analysis of triadic data contexts. How this analysis can be approached is shown by examples. Theoretically, we point out how a "Modal Attribute Logic" may be developed.
\end{abstract}

\section{Contents}

1. Conditional Modality

2. Necessity and Possibility in Triadic Contexts

3. Modal Attribute Logic

\section{Conditional Modality}

The modal understanding of propositions has been semantically based on the notion of "possible worlds". The basic idea of modal semantics is that a proposition is "necessarily true" if it is true in all (accessible) possible worlds, and "possibly true" if it is true in some (accessible) possible world (cf. [HC90]). Criticisms of this semantical foundation question the ontological status of the possible worlds: Are they real or fictitious? (cf. [Re95], [So98]) Instead of going deeper in that general question, we consider in this paper the more special case of states of affairs which allow different views of their matters. This shall contribute to the developement of a contextual logic of triadic contexts in analogy to the dyadic case described in [GW99b].

Such modal views often occur when several persons report about the same situation or event. An intriguing example for this is presented by the famous Japanese movie "Rashomon" (cf. [Ko95]). In the movie six persons - a robber, a samurai, the wife of the samurai, a woodcutter, a monk, and a vagabond report about an event during which the samurai was murdered and his wife was 
raped. The different reports show perspective views of the truth which, for each individual, has to coincide with her pride and sense of honour. In this way the spectator comes to know only possible truths where it remains open what really happened.

As another example with less differences between the individual presentations, the gospels of the four evangelists of the New Testament shall be mentioned. In this example the differences between the presentations are not caused by the individual characters of the narrators, but by the way of oral and written tradition in which the gospels originated. The four gospels gave rise to a formal representation of different relationships by a multicontext (see [Wi96]) and a triadic context (see [Bi98], [WZ99]), respectively, which allows to compare all passages of the gospels naming at least one of the disciples. In the triadic context the twelve disciples are considered as the formal objects, the thirty-six passages as the formal attributes, and the four evangelists as the formal conditions; the ternary relation of the triadic context indicates which disciple is mentioned in which passage by which evangelist.

An interesting question is: Which disciples are mentioned in which passages by all evangelists or by at least one evangelist? A complete answer is presented by the two concept lattices in Figure 1 and Figure 2 which underly the formal contexts with the disciples as objects, the passages as attributes, and the relation associating a disciple with a passage if that is done, in the first case, by all evangelists and, in the second case, by at least one evangelist. The information given by the first concept lattice might surprise: there are only seven passages (out of 36 ) in which the evangelists commonly name at least one disciple; furthermore, only four disciples are mentioned in those passages. The second concept lattice yields much richer information: six disciples - Peter, John, Thomas, Matthew, Thadaeus, and Judas - are mentioned alone in some passage, Andrew and Philip are unique with respect to the passages referring to them, James is possibly named always together with John, while Bartholomew, James Alphaeus, and Simon are only mentioned in passage 7 ("Sending out the twelve apostles"), together with all other disciples.

A more technical example where the modality is caused by different surroundings has been elaborated in the field of Security Management of Information Technology (see [BSI96], [Sö98], [B+98]). In the triadic context formalizing the data, the formal objects and attributes represent threats concerning information technology and safeguards to prevent those threats, respectively, while the formal conditions refer to IT-units such as IT-configurations, environments, and organizational set-ups. An important task of IT-security management is the derivation of concrete directives to prevent or react on damages. This presupposes a comprehensive analysis of the necessary and possible relationships between threats and safeguards with respect to a given set of IT-units. A main type of task is therefore: Determine for given sets of threats, safeguards, and IT-units the two concept lattices analogous to those of our example of the four gospels. The theoretical background for the treatment of this task will be outlined in the next section (for basic notions and results of Formal Concept Analysis see [GW99a]). 


\section{Necessity and Possiblity in Triadic Contexts}

Triadic Concept Analysis has been introduced as an extension of Formal Concept Analysis in [LW95] and [Wi95] (see also [Bi98]). It is mathematically based on the notion of a triadic context defined as a quadruple $(G, M, B, Y)$ where $G, M$, and $B$ are sets and $Y$ is a ternary relation between $G, M$, and $B$, i.e. $Y \subseteq G \times M \times B$; the elements of $G, M$, and $B$ are called (formal) objects, attributes, and conditions, respectively, and $(g, m, b) \in Y$ is read: the object $g$ has the attribute $m$ under the condition $b$. A triadic concept of a triadic context $(G, M, B, Y)$ is defined (analogously to a dyadic concept in Formal Concept Analysis) as a triple $\left(A_{1}, A_{2}, A_{3}\right)$ with $A_{1} \subseteq G, A_{2} \subseteq M$, and $A_{3} \subseteq B$ such that the triple $\left(A_{1}, A_{2}, A_{3}\right)$ is maximal with respect to component-wise set inclusion in satisfying $A_{1} \times A_{2} \times A_{3} \subseteq Y$. If $(G, M, B, Y)$ is described by a threedimensional cross table, this means that, under suitable permutations of rows, columns, and layers of the cross table, the triadic concept $\left(A_{1}, A_{2}, A_{3}\right)$ is represented by a maximal rectangular box full of crosses (for an extensive motivation of the definition of a triadic context see [LW95]). For a particular triadic concept $\mathfrak{c}:=\left(A_{1}, A_{2}, A_{3}\right)$, the components $A_{1}, A_{2}$, and $A_{3}$ are called the extent, the intent, and the modus of $\mathfrak{c}$, respectively. The set $\mathfrak{T}(\mathbb{K})$ of all triadic concepts of the triadic context $\mathbb{K}:=(G, M, B, Y)$ is structured by set inclusion considered in each of the three components of the triadic concepts. For each $i \in\{1,2,3\}$, one obtains a quasiorder $\lesssim_{i}$ and its corresponding equivalence relations $\sim_{i}$ defined by

$$
\begin{aligned}
& \left(A_{1}, A_{2}, A_{3}\right) \lesssim_{i}\left(B_{1}, B_{2}, B_{3}\right): \Longleftrightarrow A_{i} \subseteq B_{i} \quad \text { and } \\
& \left(A_{1}, A_{2}, A_{3}\right) \sim_{i}\left(B_{1}, B_{2}, B_{3}\right): \Longleftrightarrow A_{i}=B_{i} \quad(i=1,2,3)
\end{aligned}
$$

The relational structure $\underline{\mathfrak{T}}(\mathbb{K}):=\left(\mathfrak{T}(\mathbb{K}), \lesssim_{1}, \lesssim_{2}, \lesssim_{3}\right)$ is called the concept trilattice of the triadic context $\mathbb{K}$.

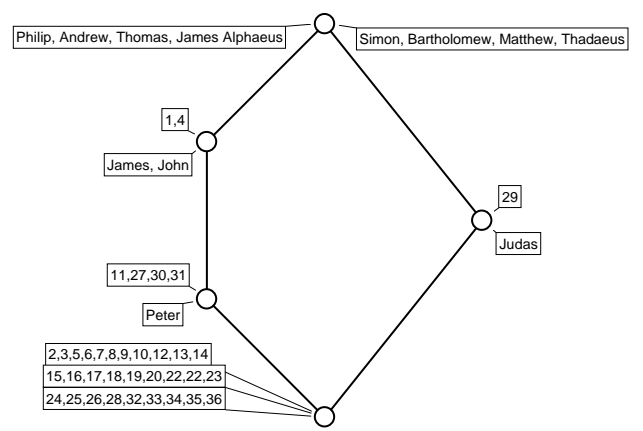

Fig. 1. The concept lattice showing which disciples are mentioned in which passages by all evangelists 


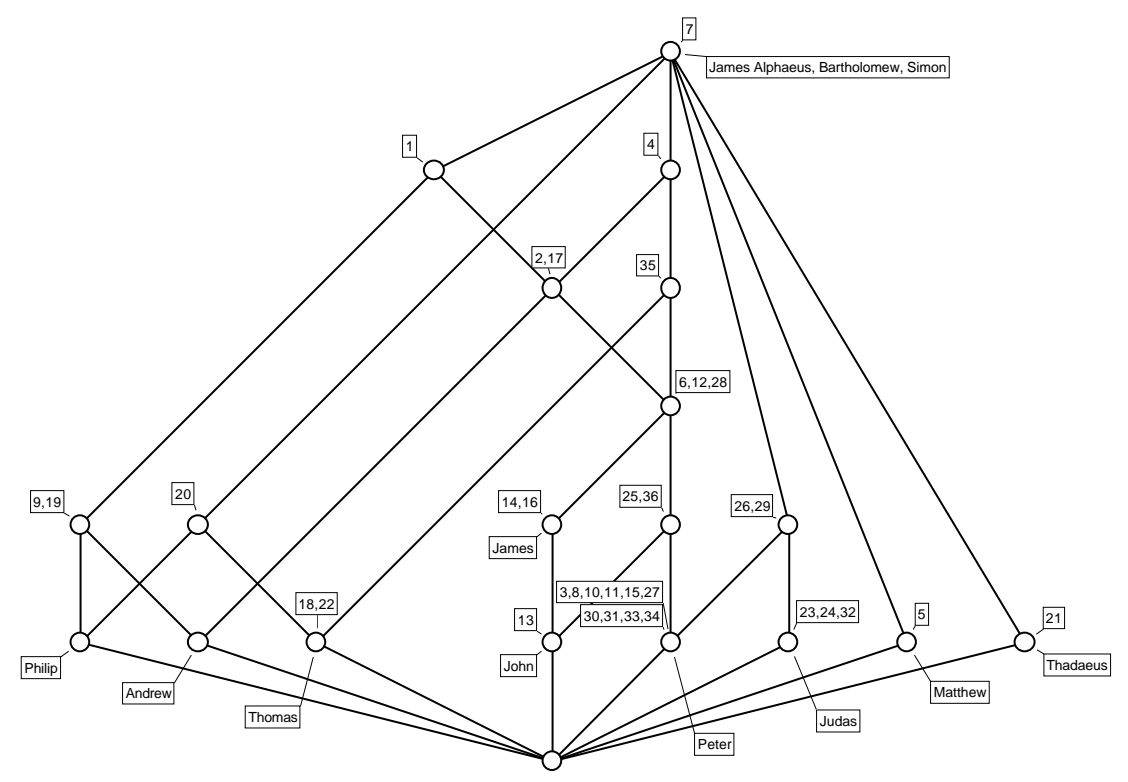

Fig. 2. The concept lattice showing which disciples are mentioned in which passages by at least one evangelist

Since, in most cases, concept trilattices of triadic contexts are very complex, it is often better to derive dyadic contexts from a triadic context and to analyse them through their dyadic concept lattices. For such derivation and subsequent analysis, the examples in Section 1 suggest the following modal approach: For a triadic context $\mathbb{K}:=(G, M, B, Y)$ the derived $\square$-context and $\diamond$-context are defined by

$$
\begin{aligned}
& \mathbb{K}_{\square}:=\left(G, M, I_{\square}\right) \text { with } g I_{\square} m: \Leftrightarrow \forall b \in B:(g, m, b) \in Y, \\
& \mathbb{K}_{\diamond}:=\left(G, M, I_{\diamond}\right) \text { with } g I_{\diamond} m: \Leftrightarrow \exists b \in B:(g, m, b) \in Y ;
\end{aligned}
$$

$g I_{\square} m$ is read: "the object $g$ has necessarily the attribute $m$ ", and $g I_{\diamond} m$ is read: "the object $g$ has possibly the attribute $m$ ". In the case $|B|=1$, which may be considered as the dyadic case, the relations $I_{\square}$ and $I_{\diamond}$ are identical, i.e. $\mathbb{K}_{\square}=\mathbb{K}_{\diamond}$. Note that each dyadic concept $(C, D)$ of the $\square$-context is part of the triadic concept $(C, D, B)$; this indicates that the concept lattice of the $\square$ context $\mathbb{K}_{\square}$ is a sublattice of the concept trilattice $\underline{\mathfrak{T}}(\mathbb{K})$. For the concept lattice of the $\diamond$-context $\mathbb{K}_{\diamond}$ there is not such a direct connection to the concept trilattice $\underline{\mathfrak{T}}(\mathbb{K})$.

The main type of task for the triadic context discussed at the end of Section 1 can now be formulated more precisely: Determine for given sets $\underline{G}$ of threats, $\underline{M}$ of safeguards, and $\underline{B}$ of IT-units the concept lattices of the $\square$-context $(\underline{G}, \underline{M}, \underline{\underline{I}} \underline{\underline{I}})$ 
and the $\diamond$-context $\left(\underline{G}, \underline{M}, \underline{I}_{\diamond}\right)$. How this can be done, we demonstrate by taking as IT-unit set $\underline{B}:=\{$ Technical Infrastructure Room, Server Room $\}$, as threat set $\underline{G}$ the threat scenario for these rooms listed in Figure 5 , and as safeguard set $\underline{M}$ the recommended countermeasures also listed in Figure 5. The two data contexts for the room of technical infrastructure (which shall be abbreviated $R T I$ ) and for the server room (which shall be abbreviated $S R$ ), both taken from [BSI96], are represented in Figure 3 and Figure 4; these two contexts form the triadic context which shall be investigated. For this, the appertaining $\square$ - and $\diamond$-context are determined together with their concept lattices which are shown in Figure 6 and Figure 7.

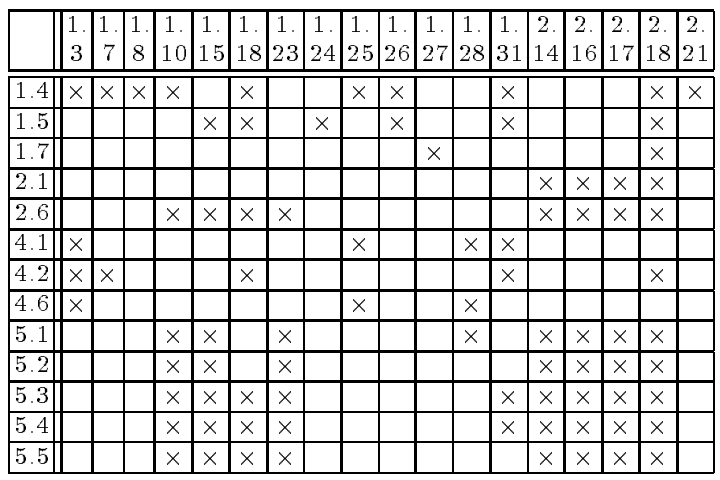

Fig. 3. Formal context of threats and safeguards concerning the serverroom (SR)

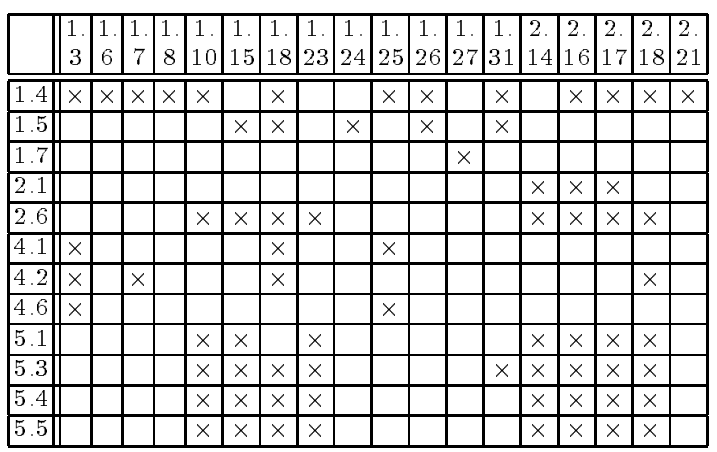

Fig.4. Formal context of threats and safeguards concerning the room for technical infrastructure (RTI) 


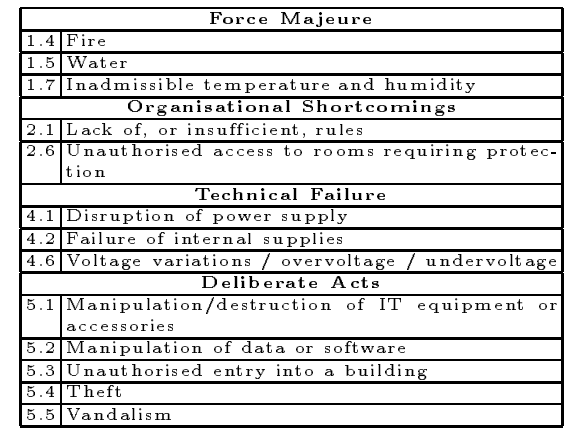

Fig. 5. Recommended countermeasures (safeguards)
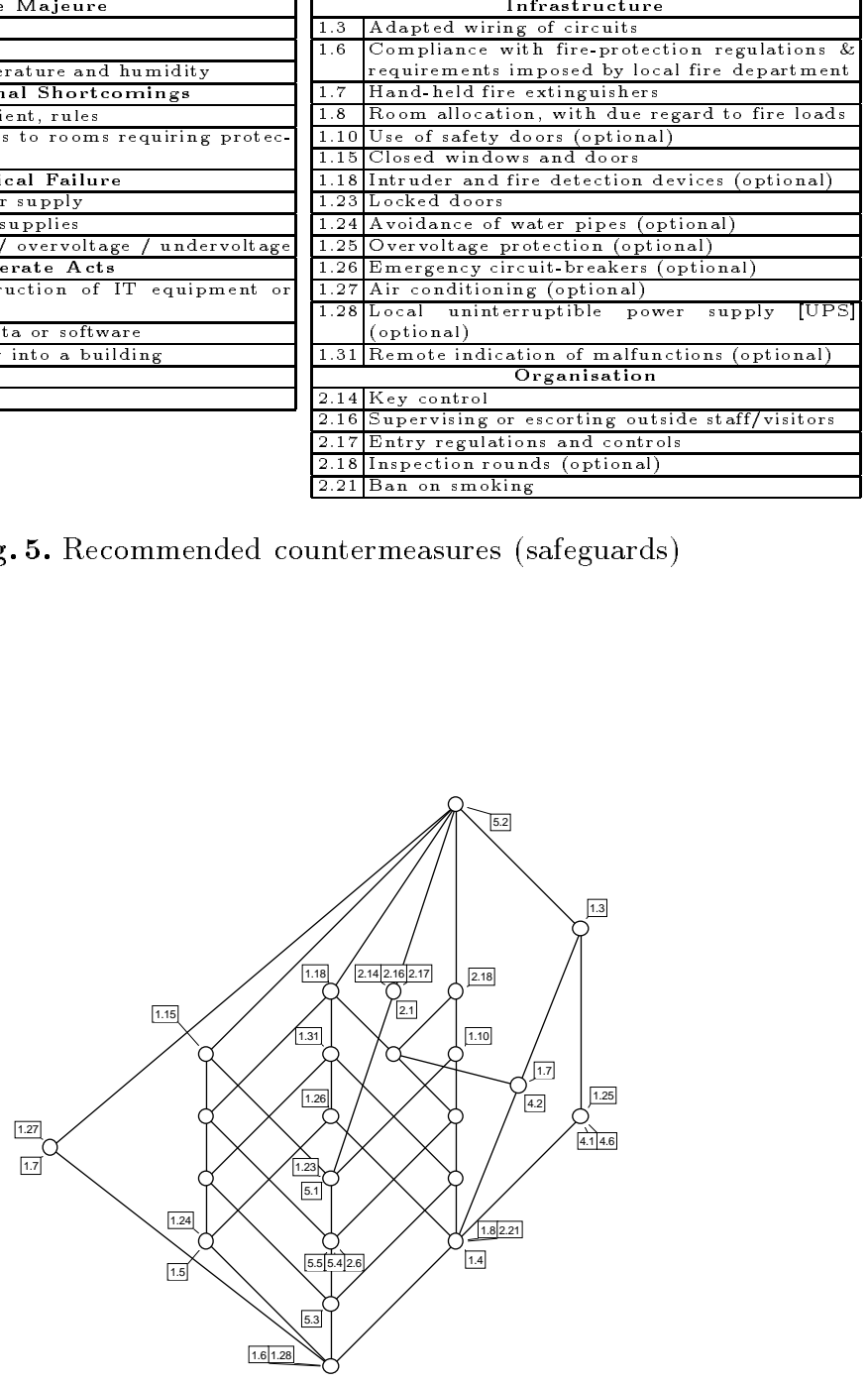

Fig. 6. The concept lattice $\mathfrak{B}\left(\mathbb{K}_{\square}\right)$ of the $\sqsubset$-context of the triadic context formed by the dyadic contexts of Figure 5 


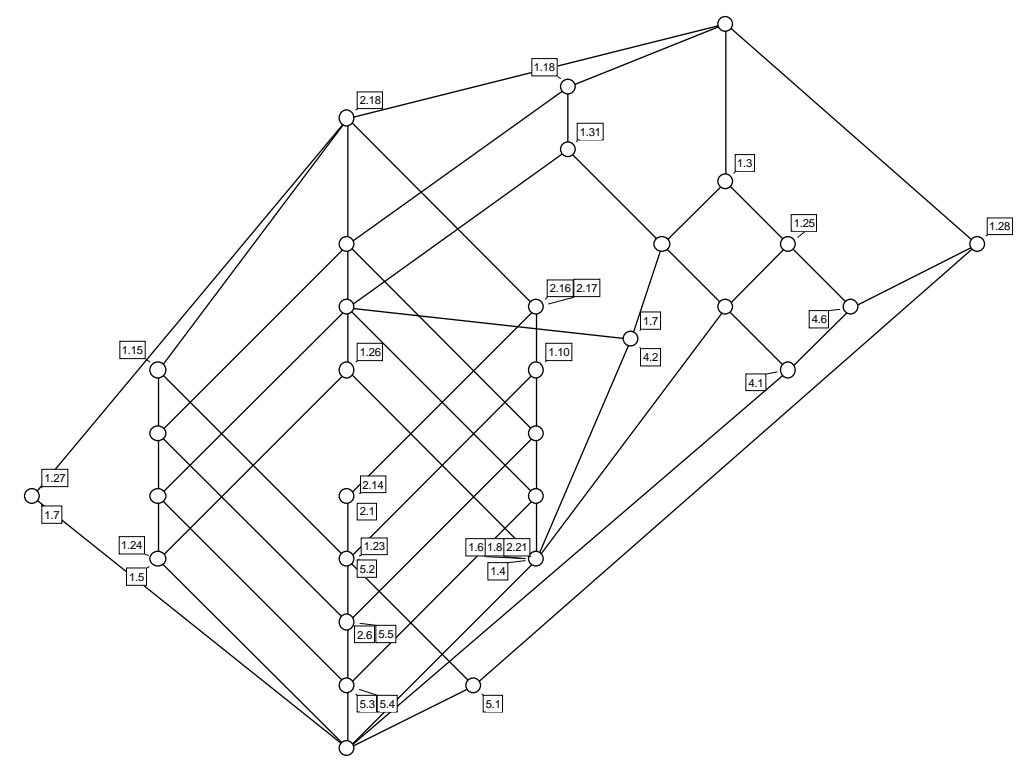

Fig. 7. The concept lattice $\mathfrak{B}\left(\mathbb{K}_{\diamond}\right)$ of the $\diamond$-context of the triadic context formed by the dyadic contexts of Figure 5

At a first glance, both diagrams look fairly similar. This is not surprising because the contexts do not differ much. Especially, the contexts are concerned with almost the same threats, except 5.2 (manipulation of data or software) which can only happen in SRs. They have also the same countermeasures, except 1.6 (compliance with fire-protection regulations) which only appears in RTIs and 1.28 (local uninterruptible power supply) which only appears in SRs. This is the reason that in $\mathfrak{B}\left(\mathbb{K}_{\square}\right)$, the top-element is labeled with the object 5.2 and the bottom-element is labeled with the attributes 1.6 and 1.28.

At a second glance, it is noticable that the right side of the concept lattice in Figure 6 looks much simpler than the right side of the the concept lattice in Figure 7. For example, the objects 4.1 (disruption of power supply) and 4.2 (failure of internal supplies) have the attribute 1.31 (remote indication of malfunction) in the $\diamond$-context, but not in the $\square$-context. This is because server provide the remote control of their functions, but in RTIs one finds in general distributors of internal supplies which usually do not provide any remote access.

Note that in the concept lattice of the $\diamond$-context, some atoms are labeled with safeguards. This means these safeguards prevent exactly one (or more) threats. For example, the countermeasure 1.24 (avoidance of water pipes) serves to prevent the threat "water". Nevertheless, this threat can be confronted with more safeguards. What is really surprising is safeguard 1.7 (Hand-held fire extinguishers). One would expect that this safeguard prevents only fire. But, as one 
can see in the concept lattice of the $\square$-context, it is always used to prevent 4.2 (failure of internal supplies). This might be a fault in the underlying contexts for SRs and RTIs.

Finally note that there is only one co-atom in the concept lattice $\mathbb{K}_{\square}$ in Figure 6 which is labeled with an object, namely 2.1 (lack of, or insufficient, rules), and there is no object-labeled co-atom in the concept lattice $\mathbb{K}_{\diamond}$ in Figure 7. This means that there exist at least two countermeasures for every threat.

\section{Modal Attribute Logic}

A first step for a systematic study of the $\square$ - and $\diamond$-context and their conceptual connections is made by investigating the "Boolean Logic of Extension" of the attributes in both contexts (cf. [GW99b],[WZ99]). For this, we introduce the logical connectives $\wedge, \vee$, and $\neg$ as partial operations on the set of attributes of any triadic context $\mathbb{K}:=(G, M, B, Y)$ for which we assume (to simplify definitions) that each attribute $m \in M$ is uniquely determined by the set $\{(g, b) \in G \times B \mid$ $(g, m, b) \in Y\}$. For $m, n, p \in M$ we define:

$$
\begin{aligned}
p=m \wedge n: \Longleftrightarrow \forall g \in G \forall b \in B:(g, p, b) \in I \Leftrightarrow(g, m, b) \in I \text { and }(g, n, b) \in I \\
p=m \vee n: \Longleftrightarrow \forall g \in G \forall b \in B:(g, p, b) \in I \Leftrightarrow(g, m, b) \in I \text { or }(g, n, w) \in I \\
p=\neg m: \Longleftrightarrow \forall g \in G \forall b \in B:(g, p, b) \in I \Leftrightarrow(g, m, b) \notin I
\end{aligned}
$$

In $\mathbb{K}_{\square}$ und $\mathbb{K}_{\diamond}$ we have the following attribute extents:

$$
\begin{aligned}
& m^{\square}:=\{g \in G \mid \forall b \in B:(g, m, b) \in Y\} \quad \text { and } \\
& m^{\diamond}:=\{g \in G \mid \exists b \in B:(g, m, b) \in Y\} \quad(m \in M)
\end{aligned}
$$

Obviously, $m^{\square} \subseteq m^{\diamond}$. Furthermore, it can be easily proved that

$(m \wedge n)^{\square}=m^{\square} \cap n^{\square},(m \vee n)^{\diamond}=m^{\diamond} \cup n^{\diamond},(\neg m)^{\square}=\left(m^{\diamond}\right)^{c}$, and $(\neg m)^{\diamond}=\left(m^{\square}\right)^{c}$

(in general, $U^{c}$ is the complement of the subset $U$ in $G$ ).

To understand which relationships between the considered attribute extents are always valid, we characterize the set systems $\mathfrak{M}(\mathbb{K}):=\left(m^{\square}, m^{\diamond}\right)_{m \in M}$ abstractly.

Proposition 1 For a set system $\mathfrak{M}:=\left(U_{m}, V_{m}\right)_{m \in M}$ there exists a triadic context $\mathbb{K}:=(G, M, B, Y)$ with $\mathfrak{M}=\mathfrak{M}(\mathbb{K})$ if and only if $U_{m} \subseteq V_{m}$ for all $m \in M$; as triadic context one can choose $\mathbb{K}_{\mathfrak{M}}:=\left(G_{\mathfrak{M}}, M,\{\square, \diamond\}, Y_{\mathfrak{M}}\right)$ with $G_{\mathfrak{M}}:=\bigcup_{m \in M} V_{m}$ and $Y_{\mathfrak{M}}:=\left\{(g, m, \square) \mid g \in U_{m}\right\} \cup\left\{(g, m, \diamond) \mid g \in V_{m}\right\}$.

For investigating the relationships between the $\square$ - and $\diamond-$ derivations and the Boolean operations, we consider now triadic contexts $\mathbb{K}:=(G, M, B, Y)$ which are Boolean-closed, i.e., the logical connectives $\wedge, \vee$, and - yield full operations on their attribute set $M$ (not properly partial); we also assume for a Boolean-closed triadic context $(G, M, B, Y)$ that each attribute $m \in M$ is uniquely determined by $m^{\square}$ (because of $m^{\square}=\left((\neg m)^{\diamond}\right)^{c}$ this implies also that it 
is also uniquely determined by $\left.m^{\diamond}\right)$. Now, the main question can be formulated: Can we understand the set sytems $\mathfrak{M}(\mathbb{K}):=\left(m^{\square}, m^{\diamond}\right)_{m \in M}$ in their role as extensional basis for a modal attribute logic as well as we understand the power sets as extensional basis for the Boolean Logic? To approach answers to this question, we show that Proposition 1 can be further elaborated for Booleanclosed triadic contexts:

Proposition 2 For a set sytem $\mathfrak{M}:=\left(U_{m}, V_{m}\right)_{m \in M}$ exists a Boolean-closed triadic context $\mathbb{K}:=(G, M, W, I)$ with $\mathfrak{M}=\mathfrak{M}(\mathbb{K})$ if and only if the following conditions hold:

1. The mappings $m \mapsto U_{m}$ and $m \mapsto V_{m}$ are injective,

2. $\left\{U_{m} \mid m \in M\right\}$ is closed under finite intersections and contains $G$,

3. $\left\{V_{m} \mid m \in M\right\}$ is closed under finite unions and contains $\emptyset$,

4. $U_{m} \subseteq V_{m}$ for all $m \in M$, and

5. There is a bijection $h: M \rightarrow M$ satisfying $\left(U_{m}\right)^{c}=V_{h(m)}$ and $\left(V_{m}\right)^{c}=$ $U_{h(m)}$ for all $m \in M$;

as Boolean-closed triadic context one can choose $\mathbb{K}_{\mathfrak{m}}$ defined in Proposition 1.

Note that, in Proposition 2, the mapping $h$ must be involutorial; furthermore, we have $\left\{V_{m} \mid m \in M\right\}=\left\{U_{m}^{c} \mid m \in M\right\}$. This leads to an abstract characterization of the set systems $\left(m^{\square}\right)_{m \in M}$ of Boolean-closed triadic contexts $(G, M, B, Y)$ :

Proposition 3 For a set system $\left(U_{m}\right)_{m \in M}$ there exists a Boolean-closed triadic context $(G, M, B, Y)$ with $U_{m}=m^{\square}$ for all $m \in M$ if and only if the mapping $m \mapsto U_{m}$ is injective, $\left\{U_{m} \mid m \in M\right\}$ is closed under finite intersections and contains $G$, and there is a bijection $h: M \rightarrow M$ being involutorial and satisfying $U_{h(m)} \cap U_{m}=\emptyset$ for all $m \in M$. Such a system shall be called $a \square$-extent system.

Note that for a $\square$-extent system $\mathfrak{U}$, the bijection $h$ is not uniquely determined. To see this, let $G:=\{1,2,3,4\}$ and $\mathfrak{U}:=(\emptyset, G,\{1\},\{1,2\},\{3\},\{3,4\})$. There are two possible mappings $h$ which satisfy the conditions of Proposition 3, scetched with the thin lines in the drawing below:
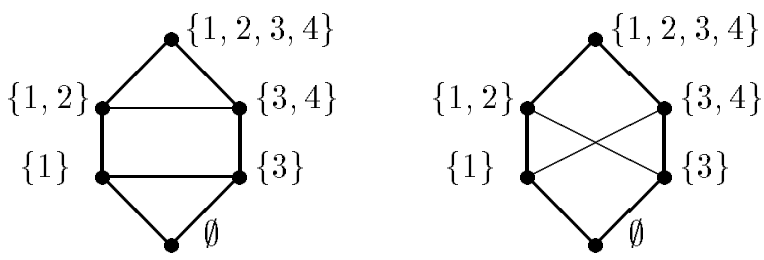

The $\square$-extent systems can be algebraically characterized as the $\wedge$-orthosemilattices which are $\wedge$-semilattices with 0 and 1 and an additional unary operation $x \mapsto x^{\perp}$ satisfying $x \wedge x^{\perp}=0$ and $x^{\perp \perp}=x$. In the rest of this section we want to examine how $\square$-extent systems can be constructed. For this we introduce the notion of an othogonal set system: 
A set system $\left(U_{m}\right)_{m \in M}$ which allows an involutorial bijection $h: M \rightarrow M$ such that $U_{h(m)} \cap U_{m}=\emptyset$ for all $m \in M$ shall be called orthogonal.

The orthogonal set systems closed under intersections are just the $\square$-extent systems. Next, we indicate some negative results concerning the construction of $\square$-extent systems:

1. The $\square$-extent subsystems of the system $\mathfrak{U}:=(\emptyset, G,\{1\},\{1,2\},\{3\},\{3,4\})$ with $G:=\{1,2,3,4\}$ do not form a closure system:

Consider $\mathfrak{V}_{1}:=(\emptyset, G,\{1\},\{3,4\})$ and $\mathfrak{V}_{2}:=(\emptyset, G,\{1\},\{3\})$. Obviously, $\mathfrak{U}$ has $\mathfrak{V}_{1}$ and $\mathfrak{V}_{2}$ as $\square$-extent subsystems, but not $\mathfrak{V}_{1} \cap \mathfrak{V}_{2}=(\emptyset, G,\{1\})$.

The question arises whether there are set systems which are easier to describe and to construct and which generate just the $\square$-extent systems. Each set system can be minimally extended to a unique set system closed under finite intersections, but not each set system is contained in a unique minimal orthogonal set system. Therefore an approach could be to start with an orthogonal set system and to close it under finite intersections. But this does not always lead to a $\square$-extent system, as the following example shows:

2. Consider the system $\mathfrak{V}:=(\emptyset, G,\{1\},\{3\},\{1,2),\{2,3\}) \cdot \mathfrak{V}$ is an orthogonal set system, but the system $\mathfrak{V}^{\prime}$ of all finite intersections with sets of $\mathfrak{V}$ is not a $\square$-extent system.

It is even worse: The $\square$-extent systems minimally extending a given set system closed under finite intersections need not only to be unique, but even not to be isomorphic:

3. In $G=\{1,2,3,4\}$, the set system $\mathfrak{V}:=(\emptyset, G,\{1\},\{1,2\})$ is closed under finite intersections and has two different $\square$-extent systems as minimal extensions which are not isomorphic, namely $\mathfrak{V}_{1}:=(\emptyset, G,\{1\},\{1,2\},\{3\},\{3,4\})$ and $\mathfrak{V}_{2}:=(\emptyset, G,\{1\},\{1,2\},\{3\},\{4\})$.
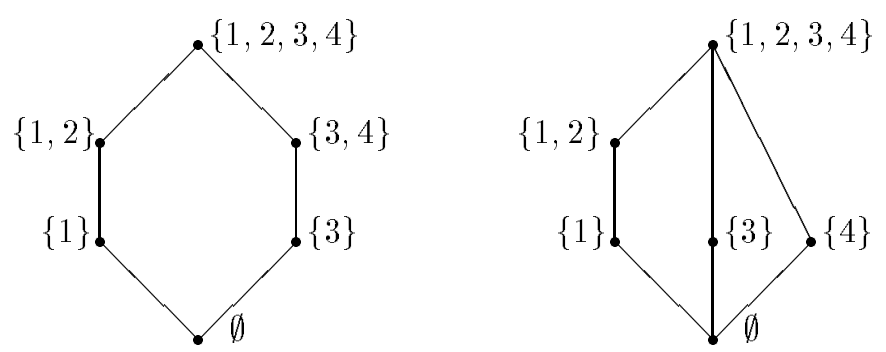

Thus, we have a description of the $\square$-extent systems, but we cannot expect to gain an easy or canonical way for constructing them. Nevertheless there is at least one interesting property: All $\square$-extent systems minimally extending a set system $\mathfrak{U}:=\left(U_{m}\right)_{m \in M}$ have the same cardinality. This is a consequence of the following proposition:

Proposition 4 Let $\mathfrak{U}:=\left(U_{m}\right)_{m \in M}$ be a set system which is closed under finite intersections, and let $\overline{\mathfrak{U}}$ be an orthogonal subsystem of $\mathfrak{U}$ with maximal cardinality. If $\mathfrak{V}$ is a $\square$-extent system minimally extending $\mathfrak{U}$, then $|\mathfrak{V}|=|\overline{\mathfrak{U}}|+2 *|\mathfrak{U} \backslash \overline{\mathfrak{U}}|$. 
The proofs of the stated propositions and further results can be found in [Da99]. But is has still to be solved how the extent systems $\left(m^{\square}, m^{\diamond}\right)_{m \in M}$ can be completely classified, analogously to the classification of the power set lattices as the completely distributive complete Boolean lattices.

\section{References}

[B+98] K. Becker, G. Stumme, R. Wille, U. Wille, M. Zickwolff: Conceptual information systems discussed through an IT-security tool. FB4-Preprint, TU Darmstadt 1998.

[Bi97] K. Biedermann: How triadic diagrams represent conceptual structures. In: D. Lukose, H. Delugach, M. Keeler, L. Searle, J. Sowa (eds.): Conceptual Structures: Fulfilling Peirce's Dream. Springer, Berlin-Heidelberg-New York 1997, 304-317.

[Bi98] K. Biedermann: A foundation of the theory of trilattices. Dissertation, TU Darmstadt 1998. Shaker Verlag, Aachen 1998.

[BSI96] Bundesamt für Sicherheit in der Informationstechnik: IT-Grundschutzhandbuch 1996. Bonn 1996.

[BT98] P. Blackburn, M. Tzakova: Hybrid Languages and Temporal Logic. CLAUSReport Nr. 107, November 1998. To appear in: Logic Journal of the IGPL

[Da99] F. Dau: Triadic Attribute Logic. (in preparation)

[GW99a] B. Ganter, R. Wille: Formal Concept Analysis: Mathematical Foundations. Springer, Berlin-Heidelberg-New York 1999.

[GW99b] B. Ganter, R. Wille: Contextual attribute logic. In: W. Tepfenhart, W. Cyre (eds.): Conceptual Structures: Standards and Practices. Lecture Notes in Artificial Intelligence 1640. Springer, Berlin-Heidelberg-New York 1999, $377-$ 388

[HC90] G. E. Hughes, M. J. Cresswell: An Introduction to Modal Logic. Routledge, London-New York 1990.

[Ko95] T. Koebner (ed.): Filmklassiker. Beschreibungen und Kommentare. Reclam, Stuttgart 1995.

[LW95] F. Lehmann, R. Wille: A triadic approach to formal concept analysis. In: G. Ellis, R. Levinson, W. Rich and J. F. Sowa (eds.): Conceptual Structures: applications, implementation and theory. Lecture Notes in Artificial Intelligence 954. Springer, Berlin-Heidelberg-New York 1995, 32-43 .

[Re95] St. Read: Thinking About Logic. Oxford University Press 1995.

[Sö98] H. Söll: Begriffliche Analyse triadischer Daten: Das IT-Grundschutzhandbuch des Bundesamts für Sicherheit in der Informationstechnik. Diplomarbeit, FB4, TU Darmstadt 1998.

[So98] J. F. Sowa: Knowledge Representation: Logical, Philosophical, and Computational Foundations. Brooks Cole Publishing Co., Pacific Grove, CA, 2000.

[Wi95] R. Wille: The basic theorem of triadic concept analysis. In: Order 12, 1995, 149-158.

[Wi96] R. Wille: Conceptual structures of multicontexts. In: P. W. Eklund, G. Ellis, G. Mann (eds.): Conceptual Structures: Knowledge Representation as Interlingua. Springer, Berlin-Heidelberg-New York 1996, 23-39.

[WZ99] R. Wille, M. Zickwolff: Grundlagen einer Triadischen Begriffsanalyse. In: G. Stumme, R. Wille (eds.): Begriffliche Wissensverarbeitung: Methoden und Anwendungen. Springer, Berlin-Heidelberg 2000, 1250 - 150 